\title{
Cognitive Speed of Processing Training Can Promote Community Mobility among Older Adults: A Brief Review
}

\author{
Melissa L. O’Connor, ${ }^{1}$ Elizabeth M. Hudak, ${ }^{2}$ and Jerri D. Edwards ${ }^{2}$ \\ ${ }^{1}$ Department of Psychology, University of Virginia, 1023 Millmont Street, Charlottesville, VA 22904, USA \\ ${ }^{2}$ School of Aging Studies, University of South Florida, Tampa, FL 33612, USA \\ Correspondence should be addressed to Melissa L.O’Connor, mocboc0516@gmail.com
}

Received 26 November 2010; Accepted 31 March 2011

Academic Editor: Lynda Anderson

Copyright (c) 2011 Melissa L. O'Connor et al. This is an open access article distributed under the Creative Commons Attribution License, which permits unrestricted use, distribution, and reproduction in any medium, provided the original work is properly cited.

\begin{abstract}
Background. Community mobility is crucial for maintaining independent functioning and quality of life for older adults. Purpose. The present paper describes the relationship of cognition, particularly speed of processing as measured by the Useful Field of View Test, to mobility as indicated by driving behaviors, life space, and falls among healthy older adults. Research examining the impact of cognitive speed of processing training (SOPT) on older adults' community mobility (i.e., driving behaviors) is also summarized. Key Issues. Even slight cognitive declines can place older adults at risk for mobility limitations. However, cognitive interventions like SOPT can mitigate declines in driving mobility. Implications. The potential of SOPT to sustain community mobility among older adults is discussed.
\end{abstract}

\section{Introduction}

Mobility may be defined as the ability to move through one's environment in order to complete a task or achieve a goal $[1,2]$. Continued mobility is crucial for maintaining independent functioning and quality of life [3, 4]. Yet, mobility limitations increase with age [3].

Decreased mobility among older adults, defined in this paper as individuals 55 years of age and older, may result from numerous factors such as failing health [3] and vision [5]. Age-related declines in aspects of cognition, including memory, reasoning, executive functioning, and speed of processing [6], may also predict mobility among communitydwelling older adults without dementia [7]. The Useful Field of View Test (UFOV, a registered trademark of Visual Awareness, Inc.) is a cognitive measure of visual processing speed for attentional tasks $[8,9]$ that has consistently emerged as a predictor of mobility. This paper describes the relationship of cognitive speed of processing, as measured by UFOV, to community mobility as indicated by driving, life space, and the occurrence of falls.

The relationship of UFOV to mobility is of particular interest in that UFOV difficulties can be rehabilitated with training [10-12]. Research has indicated that speed of processing training (SOPT), a cognitive intervention, not only improves speed of processing but also transfers to prolonged safe driving mobility among older adults $[13,14]$. Research examining the impact of this intervention on older adults' driving mobility is summarized. The potential of such cognitive interventions to sustain mobility and quality of life among older adults is discussed.

\section{Driving, Life Space, and Falls}

Driving is an important aspect of community mobility, particularly for older adults in the United States $[15,16]$. UFOV performance has been strongly connected to driving mobility outcomes in several studies $[17,18]$. For example, recent prospective studies have found that poorer UFOV performance is a significant risk factor for driving cessation, even after controlling for demographics, vision, and physical performance $[19,20]$. UFOV performance is also associated with motor vehicle crashes [21].

Life space, conceptualized as the distance that individuals move concentrically from their homes, is another distinct 
measure of community mobility $[1,22]$. Stalvey and colleagues [1] found that UFOV performance predicted life space, while visual measures did not. Wood and colleagues [7] also found that a cognitive speed factor (which included UFOV) correlated most strongly with life space as compared with other cognitive and sensory factors. Thus, although vision is important, cognitive speed of processing may be a more salient predictor of life space.

Falls are also important to consider when examining community mobility. Older adults who fall reduce their activities within the community [23] and are at higher risk for long-term care placement [24]. Factors that lead to falls include failing vision, medication use, and decreased muscle strength and flexibility $[25,26]$. Additionally, Vance et al. [27] found that cognitive decline (measured by UFOV, as well as tests of delayed recall, executive function, and visual perception) predicted higher incidence of falling among 694 older drivers.

The role of cognition in maintained mobility is of interest in that among older adults without dementia,cognitive abilities like speed of processing can be enhanced through training [28-31]. Some of the most consistent and encouraging findings of cognitive training transfer have been with a particular protocol, SOPT [32]. SOPT is a computerized, cognitive intervention that involves practice identifying and localizing visual targets at rapid display speeds (16 to $500 \mathrm{~ms}$ ) that is designed to improve UFOV. Exercise difficulty is tailored to each individual's abilities, with the overall goal of increasing the speed and accuracy of visual information processing through practice. SOPT has evolved into an exercise, RoadTour, that is a part of the training program marketed by PositScience as InSight. The program is also available through the AAA Foundation as DriveSharp. These interventions can either be completed by older adults at home on a personal computer or can be administered in settings such as independent living facilities $[33,34]$.

The efficacy of SOPT for enhancing everyday functioning and cognitive speed of processing has been demonstrated in six clinical trials among older adults [35]. Given that UFOV is strongly related to driving $[18,36]$, of particular interest was whether training could enhance driving. Searches of the terms "speed of processing training and mobility," "speed of processing training and driving," "speed of processing training and life space," and "speed of processing training and falls" were conducted in the PubMed, Ageline, PsycInfo, and Medline databases; we were interested in articles that specifically examined the SOPT protocol on domains of mobility. These searches on mobility and driving yielded 39 articles. Of these, 29 did not use SOPT and/or did not measure mobility as an outcome, and 2 described driver training programs unrelated to SOPT. Each of the remaining 8 studies found that SOPT positively enhances driving mobility among adults aged 55 and older, and these results are described below. The searches on life space and falls yielded 23 articles, none of which examined the impact of SOPT on life space or falls.

\section{Speed of Processing Training and Driving}

Roenker and colleagues [37] examined the efficacy of SOPT among adult drivers aged 55 years and older who were randomized to either SOPT or a control group of driver instruction and simulator training. Speed trained participants' UFOV scores improved an average of 2.5 standard deviations (sd) more than control group members' scores, indicating a large effect size. Speed trained participants also showed significantly improved stopping time to road signs while in a driving simulator and made $40 \%$ fewer dangerous maneuvers during an on-road driving test. These improvements were maintained over an 18-month follow-up period [37].

In the Staying Keen in Later Life (SKILL) study, older adults aged 63 years and older without dementia and with baseline UFOV difficulties were randomized to either SOPT or a social- and computer-contact control group $(n=$ 126). Training effect sizes were large and averaged $1.94 \mathrm{sd}$ of improvement in UFOV performance relative to the control group [12]. Longitudinal analyses indicated that drivers in the control group experienced greater mobility declines as evidenced by decreased driving exposure and space and increased driving difficulty over a 3-year follow-up period [14]. Drivers who completed the training program were $40 \%$ less likely than controls to cease driving during the 3 years [13].

In the Advanced Cognitive Training for Independent and Vital Elderly (ACTIVE) study [38], approximately 3,000 healthy adults aged 65 and older were randomized to one of three cognitive interventions (including SOPT) or a control group. Among the SOPT group, 87\% demonstrated reliable UFOV improvement at immediate posttest with an effect size of $1.45 \mathrm{sd}$ relative to the controls, and significant training gains endured over 5 years $[39,40]$. Older adults randomized to SOPT were about $50 \%$ less likely to experience a motorvehicle crash over the next 5 years [41].

Aside from mobility and cognitive speed of processing as measured by UFOV, SOPT also positively influences other aspects of older adults' lives. The ACTIVE study demonstrated that SOPT prevents declines in self-rated health and health-related quality-of-life across 5 years [42, 43]. Recent analyses indicate that SOPT also resulted in statistically significant reductions in predicted medical care expenditures and risk of depressive symptoms $[44,45]$. Thus, SOPT has many potential benefits for older adults.

\section{Conclusion}

Many factors impact mobility among older adults, including demographic, sensory, and medical factors [3]. However, even among community-dwelling older adults without dementia, cognition (particularly speed of processing) is independently associated with community mobility as measured by life space, falls, and driving. Even subtle cognitive declines can place older adults at risk for mobility losses.

Risk for mobility loss, particularly driving mobility, can be assessed relatively quickly with UFOV. UFOV Task 2 has been used to indicate mobility risk in a Department of Motor Vehicles-setting [18] and can be administered in 
10-15 minutes. Older adults who show UFOV difficulties (Task 2 score $\geq 150$ or Task $3+4$ score $\geq 800$ ) are most likely to immediately benefit from SOPT [35]. However, advantages from training have also been observed among general samples of older adults, including prolonged driving mobility and safety $[14,37,41]$. Furthermore, older adults have experienced maintained health-related quality of life and decreased risk for depression from participating in SOPT $[42,45]$. This testing and training technology can be implemented to promote cognitive health and sustain safe driving mobility among older adults. Although current evidence demonstrates that SOPT prolongs safe driving mobility, further research should investigate if SOPT or other cognitive interventions may preserve other aspects of community mobility as well, such as life space and falls. Since there is evidence that UFOV is related to life space and falls, these domains may be positively impacted by SOPT.

In conclusion, even among older adults without dementia, evidence-based cognitive training programs like SOPT should be considered for the goals of maintaining and possibly enhancing mobility among older adults. Such interventions have great potential to preserve independence and quality of life with advancing age.

\section{Disclosure}

Dr. Edwards has worked in the past as a limited consultant to Visual Awareness, Inc., the company that holds the patent for the UFOV assessment, and for PositScience, the company that now owns intellectual property surrounding the speed of processing training program.

\section{Acknowledgments}

The authors would like to acknowledge Stacy L. Bradley, M. A., Joan Dodson, M. A., and Jennifer Newberry of the University of Alabama at Huntsville Cognitive Aging Lab for their contributions to this paper.

\section{References}

[1] B. T. Stalvey, C. Owsley, M. E. Sloane, and K. Ball, "The life space questionnaire: a measure of the extent of mobility of older adults," Journal of Applied Gerontology, vol. 18, no. 4, pp. 460-478, 1999.

[2] G. P. Barberger and C. Fabrigoule, "Disability and cognitive impairment in the elderly," Disability and Rehabilitation, vol. 19, no. 5, pp. 175-193, 1997.

[3] J. M. Guralnik, A. Z. LaCroix, R. D. Abbott et al., "Maintaining mobility in late life. I. Demographic characteristics and chronic conditions," American Journal of Epidemiology, vol. 137, no. 8, pp. 845-857, 1993.

[4] R. A. Marottoli, L. M. Cooney, D. R. Wagner, J. Doucette, and M. E. Tinetti, "Predictors of automobile crashes and moving violations among elderly drivers," Annals of Internal Medicine, vol. 121, no. 11, pp. 842-846, 1994.

[5] C. Owsley, B. Stalvey, J. Wells, and M. E. Sloane, "Older drivers and cataract: driving habits and crash risk," The Journals of Gerontology A, vol. 54, no. 4, pp. M203-M211, 1999.
[6] H. C. Hendrie, "The NIH cognitive and emotional health project: report of the critical evaluation study committee," lzheimer's \& Dementia, vol. 2, no. 1, pp. 12-32, 2006.

[7] K. M. Wood, J. D. Edwards, O. J. Clay, V. G. Wadley, D. L. Roenker, and K. K. Ball, "Sensory and cognitive factors influencing functional ability in older adults," Gerontology, vol. 51, no. 2, pp. 131-141, 2005.

[8] J. D. Edwards, L. A. Ross, V. G. Wadley et al., "The useful field of view test: normative data for older adults," Archives of Clinical Neuropsychology, vol. 21, no. 4, pp. 275-286, 2006.

[9] J. D. Edwards, D. E. Vance, V. G. Wadley, G. M. Cissell, D. L. Roenker, and K. K. Ball, "Reliability and validity of useful field of view test scores as administered by personal computer," Journal of Clinical and Experimental Neuropsychology, vol. 27, no. 5, pp. 529-543, 2005.

[10] K. Ball, D. B. Berch, K. F. Helmers et al., "Effects of cognitive training interventions with older adults: a randomized controlled trial," Journal of the American Medical Association, vol. 288, no. 18, pp. 2271-2281, 2002.

[11] J. D. Edwards, V. G. Wadley, R. S. Myers, D. L. Roenker, G. M. Cissell, and K. K. Ball, "Transfer of a speed of processing intervention to near and far cognitive functions," Gerontology, vol. 48, no. 5, pp. 329-340, 2002.

[12] J. D. Edwards, V. G. Wadley, D. E. Vance, K. Wood, D. L. Roenker, and K. K. Ball, "The impact of speed of processing training on cognitive and everyday performance," Aging \& Mental Health, vol. 9, no. 3, pp. 262-271, 2005.

[13] J. D. Edwards, P. B. Delahunt, and H. W. Mahncke, "Cognitive speed of processing training delays driving cessation," Journals of Gerontology, vol. 64, no. 12, pp. 1262-1267, 2009.

[14] J. D. Edwards, C. Myers, L. A. Ross et al., "The longitudinal impact of cognitive speed of processing training on driving mobility," The Gerontologist, vol. 49, no. 4, pp. 485-494, 2009.

[15] D. O'Neill, "Safe mobility for older people," Reviews in Clinical Gerontology, vol. 10, no. 2, pp. 181-191, 2000.

[16] R. A. Marottoli, C. F. M. de Leon, T. A. Glass, C. S. Williams, L. M. Cooney Jr., and L. F. Berkman, "Consequences of driving cessation: decreased out-of-home activity levels," The Journals of Gerontology, vol. 55, no. 6, pp. S334-S340, 2000.

[17] G. McGwin Jr., V. Chapman, and C. Owsley, "Visual risk factors for driving difficulty among older drivers," Accident Analysis and Prevention, vol. 32, no. 6, pp. 735-744, 2000.

[18] K. K. Ball, D. L. Roenker, V. G. Wadley et al., "Can highrisk older drivers be identified through performance-based measures in a department of motor vehicles setting?" Journal of the American Geriatrics Society, vol. 54, no. 1, pp. 77-84, 2006.

[19] J. D. Edwards, E. Bart, M. L. O'Connor, and G. Cissell, "Ten years down the road: predictors of driving cessation," Gerontologist, vol. 50, no. 3, pp. 393-399, 2010.

[20] J. D. Edwards, L. A. Ross, M. L. Ackerman et al., "Longitudinal predictors of driving cessation among older adults from the ACTIVE clinical trial," Journals of Gerontology, vol. 63, no. 1, pp. P6-P12, 2008.

[21] O. J. Clay, V. G. Wadley, J. D. Edwards, D. L. Roth, D. L. Roenker, and K. K. Ball, "Cumulative meta-analysis of the relationship between useful field of view and driving performance in older adults: current and future implications," Optometry and Vision Science, vol. 82, no. 8, pp. 724-731, 2005.

[22] P. S. Baker, E. V. Bodner, and R. M. Allman, "Measuring lifespace mobility in community-dwelling older adults," Journal of the American Geriatrics Society, vol. 51, no. 11, pp. 16101614, 2003. 
[23] L. Clemson, D. Manor, and M. H. Fitzgerald, "Behavioral factors contributing to older adults falling in public places," OTJR Occupation, Participation and Health, vol. 23, no. 3, pp. 107-117, 2003.

[24] L. Mackenzie, J. Byles, and N. Higginbotham, "A prospective community-based study of falls among older people in Australia: frequency, circumstances, and consequences," OTJR Occupation, Participation and Health, vol. 22, no. 4, pp. 143152, 2002.

[25] A. J. Campbell, M. C. Robertson, M. M. Gardner, R. N. Norton, and D. M. Buchner, "Psychotropic medication withdrawal and a home-based exercise program to prevent falls: a randomized, controlled trial," Journal of the American Geriatrics Society, vol. 47, no. 7, pp. 850-853, 1999.

[26] S. R. Lord, J. Dayhew, and A. Howland, "Multifocal glasses impair edge-contrast sensitivity and depth perception and increase the risk of falls in older people," Journal of the American Geriatrics Society, vol. 50, no. 11, pp. 1760-1766, 2002.

[27] D. E. Vance, K. K. Ball, D. L. Roenker, V. G. Wadley, J. D. Edwards, and G. M. Cissell, "Predictors of falling in older Maryland drivers: a structural-equation model," Journal of Aging and Physical Activity, vol. 14, no. 3, pp. 254-269, 2006.

[28] D. X. Rasmusson, G. W. Rebok, F. W. Bylsma, and J. Brandt, "Effects of three types of memory training in normal elderly," Aging, Neuropsychology, and Cognition, vol. 6, no. 1, pp. 56-66, 1999.

[29] A. F. Kramer, J. F. Larish, and D. L. Strayer, "Training for attentional control in dual task settings: a comparison of young and older adults," Journal of Experimental Psychology, vol. 1, no. 1, pp. 50-76, 1995.

[30] K. W. Schaie, S. L. Willis, C. Hertzog, and J. E. Schulenberg, "Effects of cognitive training on primary mental ability structure," Psychology and Aging, vol. 2, no. 3, pp. 233-242, 1987.

[31] F. W. Unverzagt, D. M. Smith, G. W. Rebok et al., "The Indiana Alzheimer Disease Center's symposium on mild cognitive impairment. Cognitive training in older adults: lessons from the ACTIVE study," Current Alzheimer Research, vol. 6, no. 4, pp. 375-383, 2009.

[32] D. E. Vance, J. Dawson, V. Wadley et al., "The accelerate study: the longitudinal effect of speed of processing training on cognitive performance of older adults," Rehabilitation Psychology, vol. 52, no. 1, pp. 89-96, 2007.

[33] V. G. Wadley, R. L. Benz, K. K. Ball, D. L. Roenker, J. D. Edwards, and D. E. Vance, "Development and evaluation of home-based speed-of-processing training for older adults," Archives of Physical Medicine and Rehabilitation, vol. 87, no. 6, pp. 757-763, 2006.

[34] D. E. Vance, K. Heaton, P. L. Fazeli, and M. L. Ackerman, "Aging, speed of processing training, and everyday functioning: implications for practice and research," Activities, Adaptation and Aging, vol. 34, no. 4, pp. 276-291, 2010.

[35] K. Ball, J. D. Edwards, and L. A. Ross, "The impact of speed of processing training on cognitive and everyday functions," The Journals of Gerontology, vol. 62B, pp. 19-31, 2007.

[36] K. K. Ball, C. Owsley, B. Stalvey, D. L. Roenker, M. E. Sloane, and M. Graves, "Driving avoidance and functional impairment in older drivers," Accident Analysis and Prevention, vol. 30, no. 3, pp. 313-322, 1998.

[37] D. L. Roenker, G. M. Cissell, K. K. Ball, V. G. Wadley, and J. D. Edwards, "Speed-of-processing and driving simulator training result in improved driving performance," Human Factors, vol. 45, no. 2, pp. 218-233, 2003.
[38] J. B. Jobe, D. M. Smith, K. Ball et al., "Active: a cognitive intervention trial to promote independence in older adults," Controlled Clinical Trials, vol. 22, no. 4, pp. 453-479, 2001.

[39] S. L. Willis, S. L. Tennstedt, M. Marsiske et al., "Long-term effects of cognitive training on everyday functional outcomes in older adults," Journal of the American Medical Association, vol. 296, no. 23, pp. 2805-2814, 2006.

[40] L. A. Ross, Does speed of processing training impact driving mobility in older adults?, Ph.D. thesis, University of Alabama, Birmingham, Ala, USA, 2008.

[41] K. K. Ball, J. D. Edwards, L. A. Ross, and G. McGwin, "Cognitive training decreases motor vehicle collision involvement of older drivers," Journal of the American Geriatrics Society, vol. 58, no. 11, pp. 2107-2113, 2010.

[42] F. D. Wolinsky, F. W. Unverzagt, D. M. Smith, R. Jones, A. Stoddard, and S. L. Tennstedt, "The ACTIVE cognitive training trial and health-related quality of life: protection that lasts for 5 years," Journals of Gerontology, vol. 61, no. 12, pp. 1324-1329, 2006.

[43] F. D. Wolinsky, H. Mahncke, M. W. Vander Weg et al., "Speed of processing training protects self-rated health in older adults: enduring effects observed in the multi-site ACTIVE randomized controlled trial," International Psychogeriatrics, vol. 22, no. 3, pp. 470-478, 2010.

[44] F. D. Wolinsky, H. W. Mahncke, M. Kosinski et al., "The ACTIVE cognitive training trial and predicted medical expenditures," in Proceedings of the Transportation Research Board Annual Meeting, National Acadamies of Science, Washington, DC, USA, 2009.

[45] F. D. Wolinsky, M. W. Vander Weg, R. Martin et al., "The effect of speed-of-processing training on depressive symptoms in ACTIVE," Journals of Gerontology, vol. 64A, no. 4, pp. 468472, 2009. 


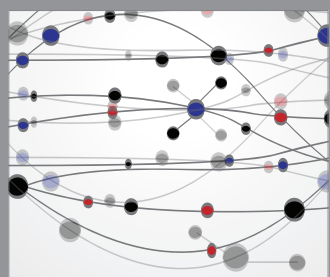

The Scientific World Journal
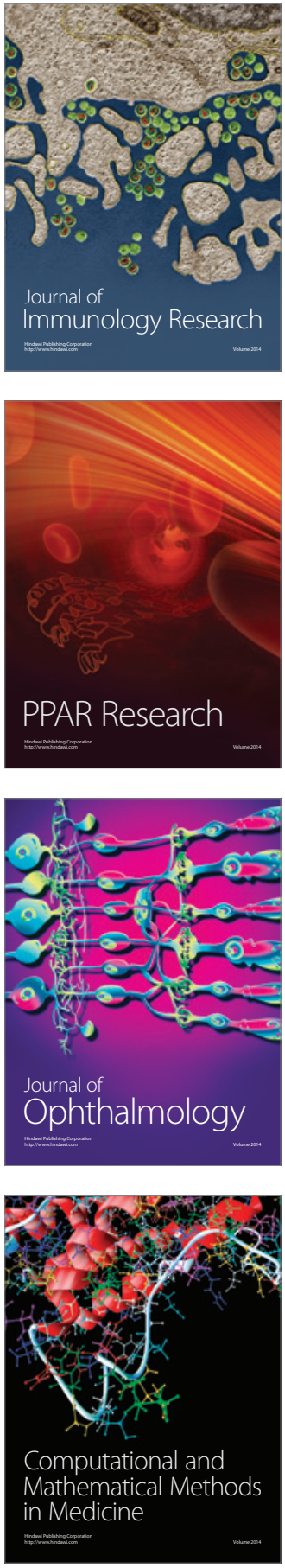

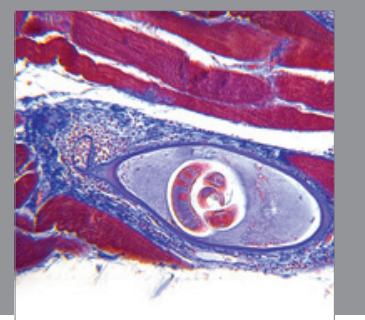

Gastroenterology

Research and Practice
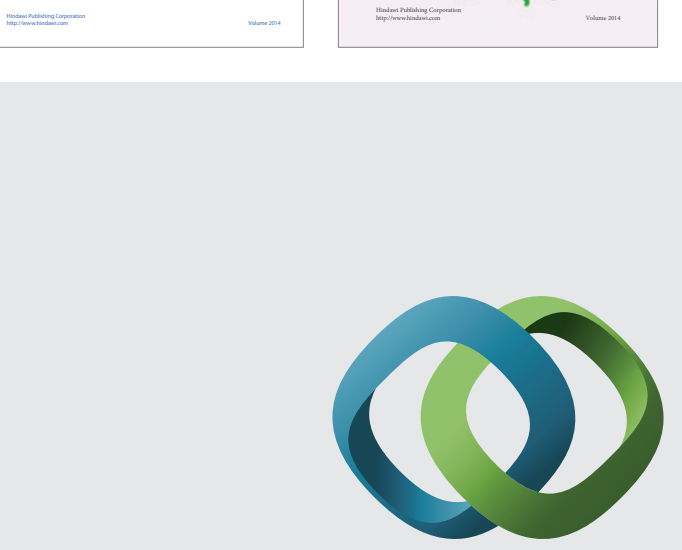

\section{Hindawi}

Submit your manuscripts at

http://www.hindawi.com
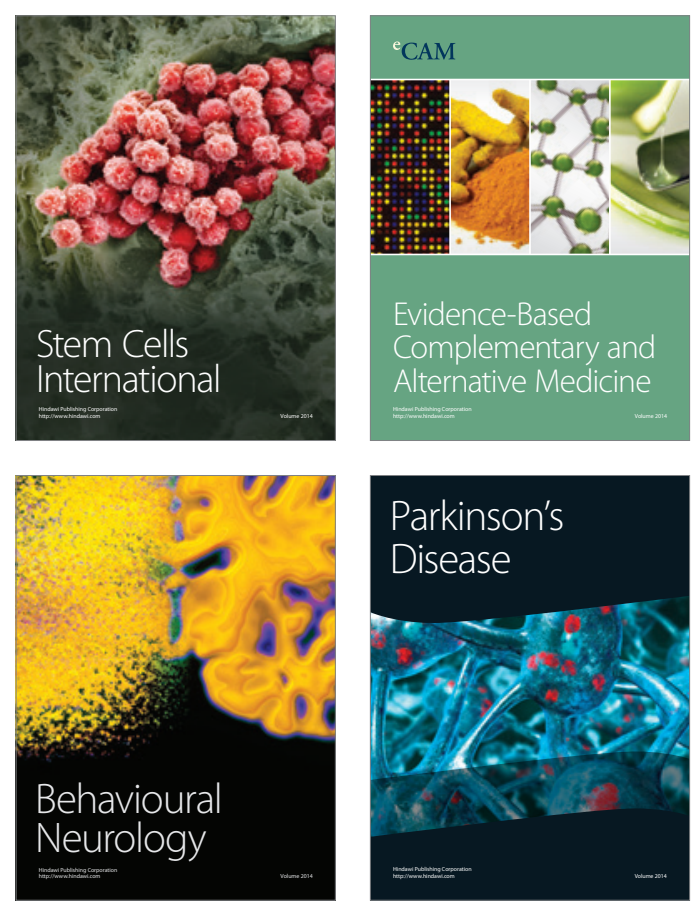

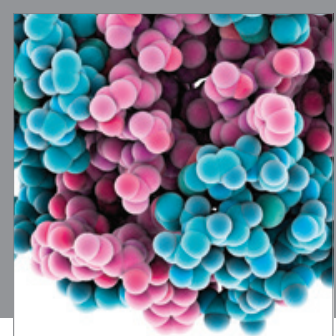

Journal of
Diabetes Research

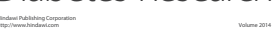

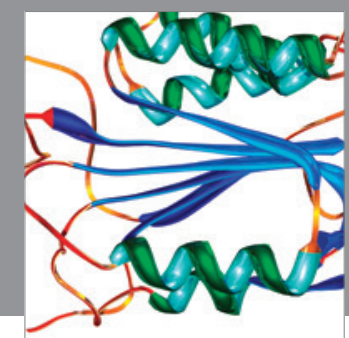

Disease Markers
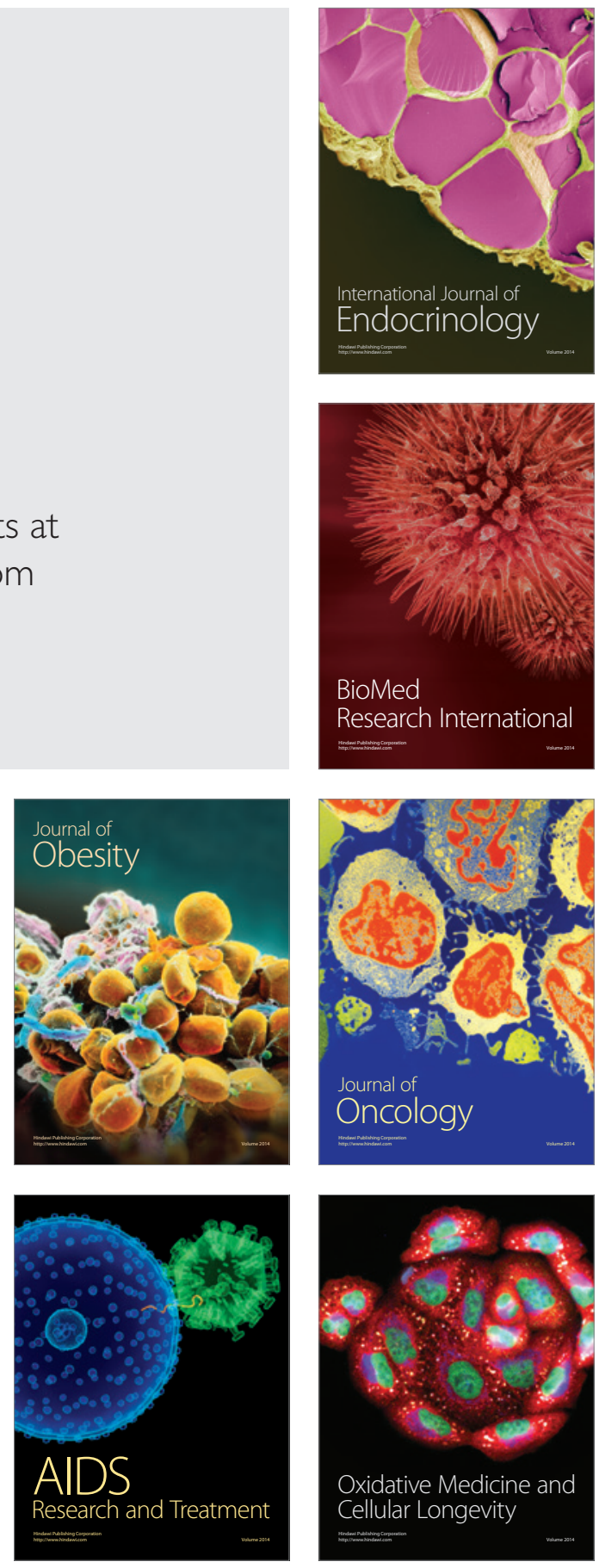